\title{
Agravamiento de enfermedades crónicas en el contexto del COVID-19: Implicación en la salud oral
}

\author{
Aggravation of noncommunicable diseases in the context of COVID-19: Implication on oral health \\ Luis Antonio Vicuña-Huaqui ${ }^{1, a}$, Francisca Alejandra Riquelme-Arenas ${ }^{2, a}$ ， Wislea Silva-Queiroz ${ }^{2, a}$
}

\section{Sr. Editor.}

El desarrollo económico de las últimas décadas conllevó una transición epidemiológica a enfermedades no transmisibles (ENT), estas presentan factores de riesgo (1), como la dieta no saludable, inactividad física, tabaco, contaminación aérea, presión sanguínea alta, obesidad, depresión, colesterol elevado y exceso de alcohol (2), que conllevan a condiciones como cáncer, enfermedades respiratorias, diabetes II y enfermedad cardiovascular que representan el $80 \%$ de muertes por ENT; el tratamiento de estas condiciones requiere el empleo de ingresos propios, $48 \%, 36 \%$ y $15 \%$ en países de renta baja, media y alta, respectivamente y el gasto sanitario estatal de $75 \%$ y $97 \%$ en países de renta baja y media-baja, respectivamente (3).

El desarrollo de la COVID-19 conllevó a la interrupción total o parcial de servicios ambulatorios de prevención y tratamiento de ENT por la reasignación de personal a áreas COVID-19, interrupción de cadenas de suministro de distribución de medicamentos y productos sanitarios, cancelación de tratamientos planificados e inasistencia de pacientes por reducción del trasporte y el temor de contagio (4); además las cuarentenas y el aislamiento social favorecieron efectos negativos en la salud mental como angustia, tristeza, ira, rabia, aislamiento, insomnio y baja autoestima, trastornos como depresión, ansiedad, trastorno obsesivo-compulsivo, estrés postraumático y suicidio (5), estas situaciones implican la pérdida de control de muchos factores de riesgo y posibles complicaciones y/o muerte por las ENT.

Las ENT se vinculan de manera estrecha a patologías bucales e inducen a un mayor desarrollo de lesiones de caries dental, enfermedades periodontales y cáncer bucal (6), ambas condiciones comparten muchos factores de riesgo, acrecentados en este nuevo contexto sanitario, como casos de trastornos alimenticios y la reducción de la higiene bucal, asociado en muchos casos por la pérdida de rutinas laborales o académicas (7), como consecuencia de la menor necesidad de contacto con otras personas, la higiene oral deficiente 
conlleva al riesgo de desarrollar de enfermedades bucales que generen dolor, sepsis, pérdida de la calidad de vida, ausentismo escolar, trastornos familiares, reducción la productividad laboral, etc.(8); las condiciones mentales, antes descritas, favorecer al desarrollo de lesiones no cariosas como la abfracción, abrasión, atrición y bruxismo y efectos a nivel neuromuscular y de la articulación témporomandibular como el trismus, a nivel de las mucosas el desarrollo de estomatitis aftosa, liquen plano oral, herpes simple recidivante, lengua geográfica, síndrome de boca ardiente (9). El tratamiento de estas condiciones conllevan a elevados gastos familiares, de difícil acceso para personas de escasos recursos, y los gastos estatales en odontología representan solo de $5-10 \%$ del presupuesto sanitario total, siendo la caries la cuarta patología más cara de tratar (6), aunado a estas dificultades se presentan restricciones del empleo de instrumentales rotatorios, reducción de aforo y la sola atención de urgencia y emergencias conllevan a un círculo vicioso de relación entre las ENT y las enfermedades bucales (10).

Conflicto de intereses: Los autores no tienen conflicto de interés con este informe.

Financiamiento: Ninguno.

Contribuciones de los autores: Todos los autores contribuyeron a este manuscrito.

\section{Correspondencia:}

Luis Antonio Vicuña Huaqui

Psj. Pucará Mz. F Lt. 2 A. H. Andrés A. Cáceres Sect. Andrés Cáceres

San Juan de Lurigancho,Lima,Perú.

Correo electrónico: Luis.vicuna1@unmsm.edu.pe

\section{REFERENCIAS BIBLIOGRAFICAS}

1. Dye BA. The Global Burden of Oral Disease: Research and Public Health Significance. J Dent Res. 2017;96(4):361-3.
2. Peters R, Ee N, Peters J, et al. Common risk factors for major noncommunicable disease, a systematic overview of reviews and commentary: the implied potential for targeted risk reduction. Ther Adv Chronic Dis. 2019;10:2040622319880392.

3. Allen LN. Financing national non-communicable disease responses. Glob Health Action. 2017;10(1): 1326687.

4. Organización de las Naciones Unidas. Political declaration of the High-level Meeting of the General Assembly on the Prevention and Control of Noncommunicable Diseases. Washington DC: Organización de las Naciones Unidas Naciones Unidas; 2011. (Citado el 25 de junio de 2021). Disponible en: https:// digitallibrary.un.org/record/710899

5. Larios-Navarro A, Bohórquez-Rivero J, NaranjoBohórquez J, Sáenz-López J. Impacto psicológico del aislamiento social en el paciente comórbido: a propósito de la pandemia COVID-19. Rev Colomb Psiquiatr. 2020;49(4):227-8.

6. Heilmann A, Tsakos G, Watt R. Oral Health Over the Life Course En: Burton-Jeangros C, Cullati S, Sacker A, et al., editors. A Life Course Perspective on Health Trajectories and Transitions. Nueva York: Springer; 2015. (Citado el 25 de junio de 2021). Disponible en: https://www.ncbi.nlm.nih.gov/books/NBK385369/

7. Zhang S, Liu C, Zhang C, Jiang H, Tai B, Du M. Impact of COVID-19 on the oral health of adults in Wuhan and China: results of a nationwide online cross-sectional questionnaire survey. BMC Oral Health. 2021; 21(1):162.

8. Peres MA, Macpherson LMD, Weyant RJ, Daly B, Venturelli R, Mathur MR, et al. Oral diseases: a global public health challenge. Lancet. 2019;394(10194):24960.

9. Luciano R, Gésime J. Condiciones bucales y su relación con el estrés. Revisión actualizada. Acta odontológica venezolana. 2016;54(2):1-9.

10. Riad A, Boccuzzi M, Pold A, Krsek M. The alarming burden of non-communicable diseases in COVID-19 new normal: Implications on oral health. Oral Dis. 2021;27(S3):791-2. 